\title{
Web Personalization based on Usage Mining
}

\author{
Sharhida Zawani Saad \\ School of Computer Science and Electronic Engineering, \\ University of Essex, \\ Wivenhoe Park, Colchester, Essex, CO4 3SQ, UK \\ szsaad@essex.ac.uk
}

\begin{abstract}
Personalized or recommender systems are a particular type of information filtering applications. User profiles, representing the information needs and preferences of users, can be inferred from log or clickthrough data, or the ratings that users provide on information items, through their interactions with a system. Such user profiles have been used, for example in iGoogle, to provide personalized recommendations to the users. A user model is a representation of this profile, which can be obtained implicitly through the application of web usage mining techniques.

Our work aims to develop Web usage mining tasks to model an intranet or local Web site recommender system. We will focus on the users activity on a university Web site, to customize the contents and structure the presentation of a Web site according to the preferences derived from the user's activity. The customization is based on an individual's user profile as well as a profile representing the collective interest of the entire user community, in this case all users accessing the Web site. The outcome will be personalized recommendations and presentation of a Web site with respect to the user's needs.
\end{abstract}

Keywords: Web usage mining, Recommender systems, Adaptive Web sites, Personalization

\section{INTRODUCTION}

The explosive growth of the Web has triggered an increasing demand of Web personalization systems. Personalized information technology services have become a ubiquitous phenomenon, taking advantage of the knowledge acquired from the analysis of the user's navigational behavior or usage data. Web usage mining (WUM) aims at discovering interesting patterns of use by analyzing web usage data. This method provides an approach to the collection and pre-processing of those data, and the construction of models representing the behavior and the interests of users. These models can be automatically incorporated into personalization components, without the intervention of any human expert (Girardi and Marinho, 2007).

Past research only focuses on how to meet consumers' information needs from the perspective of functional information, and they exclude most of the other information needs from their consideration (France et al., 2002). Research conducted by France et al. stated that the attempts of search engines and data mining technology to improve Web information search capabilities to match up various information needs has been limited. Our research aims to work on usage mining techniques to provide a user with personalized recommendations, by customizing the contents of a Web site with respect to the user's needs.

Let us use a university Web site as an example. Every user of that Web site (be it a student, a member of staff or an external visitor) will have different interests, the challenge is to tailor the presentation of the document collection according to each of these user's profiles. Obviously, this will only work if the user is in fact interested in having a personalised Web site. If not, then no user activity will be recorded and no personalisation takes place. The Web site appears unaltered. 
While each user has individual interests, we can expect a lot of overlap. A newly registered student who is searching for the teaching timetable is not alone. A lot of other students share this information need and will have searched (and hopefully located) the appropriate documents on the Web site. Hence, a Web site presented according to the entire user community's information access activities is likely to make it easier for new users to find relevant documents quickly. Again, there is no need to enforce such customization, this can easily be switched off and the Web site appears as normal.

The point of the above example is that we are hoping to capture both user and community search/navigation trails to make the entire Web site adaptive and customize it according to the two profiles which capture these activities: user and community profiles.

Originally, the aim of Web usage mining has been to support the human decision making process (Baraglia and Silvestri, 2007). Thus, the outcome of the process is typically a set of data models that reveal knowledge about usage patterns of users. WUM typically extracts knowledge by analyzing historical data such as Web server access logs, browser caches, or proxy logs. Using WUM techniques, it is possible to model user behavior, and therefore, to forecast their future movements (Baraglia and Silvestri, 2007). The information mined can subsequently be used in order to personalize the contents of Web pages. Web personalization (WP) or recommender systems are typical applications of WUM. Although most of the work in Web usage mining is not concerned with personalization, its relationship to personalization issues has brought promising results (Girardi and Marinho, 2007). The knowledge discovered through the usage mining process serves as operational knowledge to personalization systems (Girardi and Marinho, 2007). Realizing the potential of WUM techniques to construct this knowledge, our proposed research aims to provide the personalization recommendations to the users, as an output from analyzing the user's navigational behavior or usage data.

\section{RESEARCH QUESTIONS AND OBJECTIVES}

We are conducting research designed to answer the following questions:

- How can Web usage mining techniques capture the behaviour and interests of users of a Web site?

- How can the captured knowledge be utilized to construct personalized recommendations on the content and presentation of the Web site?

The following objectives have been formulated to answer the research questions:

- To develop Web usage mining tasks based on some phases and applied techniques such as data collection, data pre-processing, pattern discovery and knowledge post-processing

- To construct models representing the behavior and the interests of individual users as well as a community of users from local Web sites or intranets

- To construct an integrated profile for any related patterns of profile based on the user and community profiles

- To construct personalized presentations guided by profiles (based on the integrated profiles).

\section{RELATED WORK}

Relevant work and field studies related to Web usage mining and personalization are discussed. Among the important subjects being discussed are adaptive Web sites, Web usage mining, recommender systems, and personalization, its previous work and how they relate to our research.

\subsection{Adaptive Web Sites}

Adaptive web sites automatically improve their organization and presentation by learning from user access patterns (Perkowitz and Etzioni, 1997). There is a lot of work going on about mining 
the users' clickthrough patterns and understanding query intent to improve search result sets, e.g. (Hu et al., 2009) and (Bayir et al., 2009). Adaptive web sites can make popular pages more accessible, highlight interesting links, connect related pages, and cluster similar documents together. Perkowitz and Etzioni discuss possible approaches to this task and how to evaluate the community's progress. The focus is either on customization: modifying web pages/site's presentation in real time to suit the needs of individual users; or optimization: altering the site itself to make navigation easier for all. One user's customization does not apply to other users; there is no sharing or aggregation of information across multiple user, and transformation has the potential to overcome both limitations (Perkowitz and Etzioni, 2000). In the Web domain, we observe a visitor's navigation and try to determine what page she is seeking, to offer the desired page immediately. Index page synthesis is a step towards the long-term goal of change in view: adaptive sites that automatically suggest reorganizations of their contents based on visitor access patterns (Perkowitz and Etzioni, 1999).

Our research will be focusing on customization: to modify Web pages/site's presentation to suit the needs of individual users. However, this will be based on the integrated profile, which combine both the user profile and community profile. Perkowitz and Etzioni introduce transformation as their approach, while our work will be using both customization (based on the user profile), and also transformation (which is related to the community profile). Compared to Perkowitz and Etzioni's work, this work will be fully automatic and does not involve any Web administrator / webmaster within the process. Apart from that, their previous work and motivation focus much on index page synthesis, and aims to automate the placement of new index pages at the Web site. Their work are not focusing on user profiles and they are looking for adaptive sites as the long-term goal of the research, while this work is focusing on adaptive system and personalization based on the user and community profiles. Compared to other previous works, the main difference is to adapt its content and presentation based on the integrated profile, for local Web site or intranet access. Apart from that, the system will not be relying on explicit user feedbacks or working with any webmaster control.

\subsection{Web Usage Mining}

During the last years, researchers have proposed a new unifying area for all methods that apply data mining to Web data, named Web mining (Kosala and Blockeel, 2000). Web mining is traditionally classified into three main categories: Web content mining, Web usage mining, and Web structure mining. Web usage mining aims at discovering interesting patterns of use by analyzing Web usage data (Girardi and Marinho, 2007). It is the process of applying data mining techniques to the discovery of usage patterns from Web data generated by user interactions with a Web server, including Web logs, clickstreams and database transactions at a Web site or at a group of related sites (Corsini and Marcelloni, 2006). (Kruschwitz et al., 2008) suggest to apply log analysis technique to electronic document collections and intranets, as search in this type of collections has attracted much less attention, but locating information within such collections can be as difficult as the open Web. Web usage mining is an excellent approach to make dynamic recommendations to a Web user, based on his/her profile in addition to usage behaviour. Usage patterns extracted from Web data have been applied to a wide range of applications (Srivastava et al., 2000).

Different modes of usage or mass user profiles can be discovered using Web usage mining techniques that can automatically extract frequent access patterns from the history of previous user clickstreams stored in Web log files (Nasraoui et al., 2008). These profiles can later be harnessed towards personalizing the Web site to the user. The issue of Web mining is discussed in detail in (Eirinaki and Vazirgiannis, 2003). Apart from Web usage mining, user profiling techniques can be performed to form a complete customer profile (Eirinaki and Vazirgiannis, 2003). Our research aims to model personalization components based on Web usage mining techniques. Generally, the outcome would be the model of Web personalization systems that elaborate the Web usage information based on user's navigational behavior. The following section discusses Web recommender and personalization systems, and how they relate to our research. 


\subsection{Recommender Systems}

Collaborative filtering and content-based filtering are two types of common recommender systems. Collaborative filtering (CF) is the process of filtering or evaluating items through the opinions of other people (Schafer et al., 2007). Ratings in a collaborative filtering system may be gathered through explicit or implicit means, or both. According to Schafer et al., collaborative filtering can predict what information users are likely to want to see, enabling providers to select subsets of information to display. In this way, collaborative filtering enables the Web to adapt to each individual user's needs. Content-based recommendation systems analyze item descriptions to identify items that are of particular interest to the user. A profile of the user's interests is used by most recommendation systems. (Pazzani and Billsus, 2007) describe two types of information for user profiles; a model of the user's preferences and a history of the user's interactions with the system. One important use of the history is to serve as training data for a machine learning algorithm that creates a user model. When the user explicitly rates items, there is little or no noise in the training data, but users tend to provide feedback on only a small percentage of the items they interact with.

Content-based filtering and collaborative filtering have long been viewed as complementary. Content-based filtering can predict relevance for items without ratings, while collaborative filtering needs ratings for an item in order to predict for it. Content-based filtering needs content to analyze, and collaborative filtering does not require content. While people find the quality of multimedia data (e.g., images, video, or audio) for web pages important, it is difficult to automatically extract this information (Schafer et al., 2007). More often collaborative filtering and content-based filtering are automatically combined, sometimes called a hybrid approach. Such systems generally use CF to try and capture features like quality that are hard to analyze automatically. Our research aims to provide personalized navigational information that elaborates the web usage information based on individual user and user community profiles. This could be done based on the user's activity or interaction through emails, searching or navigation behaviour. In this case, collaborative filtering may not be necessary apart from content-based filtering, to capture important usage patterns. The research direction is more into providing personalized recommendations by identifying the area of interests of a user, and to adapt with the community profile.

\subsection{Personalization}

Web usage mining has been suggested as a new generation of personalization tools which can address the shortcomings of more traditional systems such as manual decision rule systems, collaborative filtering systems and content-based filtering agents (Mobasher et al., 2000). The idea of Mobasher et al. is to use page view and session information to automatically obtain usage profiles. The WebPersonalizer System is presented, which uses two methods to discover usage profiles: computing session clusters and association rule discovery. Research done by (Albayrak et al., 2005) propose a multi-agent system composed of four classes of agents: many information extracting agents, agents that implement different filtering strategies, agents for providing different kinds of presentation and one personal agent for each user. The personal agent should constantly improve the knowledge about "his" user by learning from the given feedback, which is taken for collaborative filtering. (Teevan et al., 2005) suggest to re-rank the results provided by current search engines. They mention that implicit user feedback is more useful compared to explicit feedback. Our research is not going to personalize the results provided by search engines, but will focus on a personalized presentation of the Web site.

(Agichtein et al., 2006) introduce robust, probabilistic techniques for interpreting clickthrough evidence by aggregating across users and queries. They interpret post-search user behaviour to estimate user preferences in a real Web search setting. They show that by aggregating user clickthroughs across queries and users, they achieve higher accuracy on predicting user preferences. Agichtein et al. aim to improve Web search ranking. Our research is not looking at Web search scenarios, but at intranet search. Besides that, Agichtein et al. use queries from query logs (from major Web search engines) with search results that were manually rated, and user interaction data. Differently, our research will be based on the user's profile and community profile 


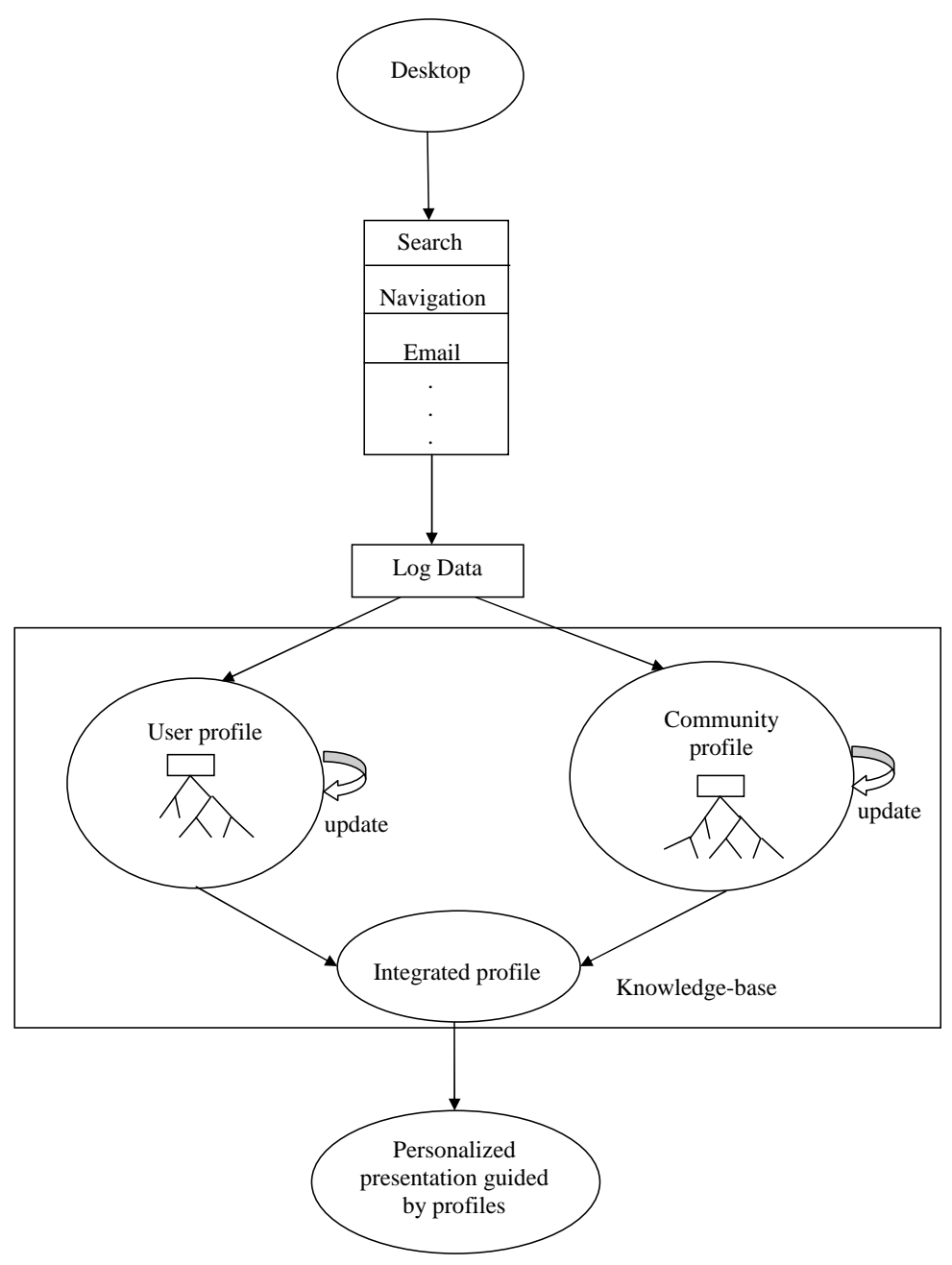

FIGURE 1: Preliminary architecture

to provide personalized recommendations, which will be done automatically and does not involve any manually rated results. A number of systems for personal search are available on today's PCs, including systems from Microsoft (desktop.msn.com), and Google (desktop.google.com). Research by (Cutrell et al., 2006) suggest Phlat, that combines keyword and property-value search, allowing users to find information based on whatever they may remember. Among problems and research directions being addressed is whether these designs can be extended to include 'non-personal' content (information sources of interest that users are not familiar with, such as news and intranet). Three research areas to be explored in personalization include recommendations, information filtering, and personalized presentation. These three areas are relevant but fairly big, therefore our future work will look into personalized presentation. This is to be done based on the user's profile from his searching/navigation activities, in combination with the community profile. 


\section{RESEARCH OUTLINE, DATA STRUCTURE AND EVALUATION}

The system's preliminary architecture and some relevant data structures is shown in figure 1 . The data flow is as follows:

- A user's activity is logged based on emails, searching and navigation behaviour.

- Web log analysis techniques are used to trace a user's behaviour or preferences.

- All the user's activities will be used to construct a user profile. The community profile is built in the same way without restricting it to a single user.

- An integrated profile combines user and community profiles in a uniform way.

- The outcome is a personalized presentation guided by profiles (based on the integrated profile).

Different types of data structure from Web page, email, text document and image can be treated in a uniform way. Each of them can be broken down into entities like title, descriptor or meta tags, content, unique identifier and contextual information. For example, contextual information of a Web page include Web documents under the same URL, while contextual information of an email include email of the same thread or information whether the email was read or written by the user.

We are at a very early stage of this research but we imagine that the outcomes will be useful in a number of different information access tasks including search, browsing and navigation. Ultimately, we are aiming at evaluating the developed techniques in some realistic Intranet settings and compare them against sensible baselines, e.g. standard search interfaces. In more detail, we plan to conduct task-based evaluations involving real users of a university Web site. The evaluations will have to address the questions whether information can be found in fewer interaction steps when using profiles, whether more relevant information can be found, whether the comparison of the two approaches (personalized versus baseline) shows differences in user satisfaction, etc. As a preliminary guide we use the evaluation paradigms developed for the TREC Interactive Track.

We do appreciate that one problem of such personalized search is the issue of "consistency", i.e. users expect consistency in a system's behaviour. We will need to address this issue and include some appropriate measures in the evaluation process.

\section{ACKNOWLEDGEMENTS}

I would like to thank my supervisor Udo Kruschwitz for his help, advice and helpful feedback on drafting this paper, and two anonymous reviewers for their helpful feedback on an earlier version of this document.

\section{References}

Agichtein, E., Brill, E., Dumais, S. T. and Ragno, R. (2006), 'Learning user interaction models for predicting web search result preferences', Personal Information Management pp. 251-259.

Albayrak, S., Wollny, S., Varone, N., Lommatzsch, A. and Milosevic, D. (2005), 'Agent technology for personalized information filtering: The pia-system', ACM Symposium on Applied Computing 1(1), 54-59.

Baraglia, R. and Silvestri, F. (2007), 'Dynamic personalization of web sites without user intervention', Communications of The ACM 50(2), 63-67.

Bayir, M. A., Toroslu, I. H., Cosar, A. and Fidan, G. (2009), Smart miner: A new framework for mining large scale web usage data, in 'Proceedings of WWW 2009', ACM, pp. 161-170.

Corsini, P. and Marcelloni, F. (2006), 'A fuzzy system for profiling web portals users from web access log', Journal of Intelligent and Fuzzy Systems 17(1), 503-516.

Cutrell, E., Robbins, D. C., Dumais, S. T. and Sarin, R. (2006), 'Fast, flexible filtering with phlat personal search and organization made easy', Personal Information Management 1, 261-269. 
Eirinaki, M. and Vazirgiannis, M. (2003), 'Web mining for web personalization', ACM Transactions on Internet Technology 3(1), 1-27.

France, T., Yen, D., Wang, J.-C. and Chang, C. M. (2002), 'Integrating search engines with data mining for customer-oriented information search', Information Management and Computer Security 10(5), 242-254.

Girardi, R. and Marinho, L. B. (2007), 'A domain model of web recommender systems based on usage mining and collaborative filtering', Requirements Eng 12(1), 23-40.

Hu, J., Wang, G., Lochovsky, F., Sun, J.-T. and Chen, Z. (2009), Understanding users query intent with wikipedia, in 'Proceedings of WWW 2009', ACM, pp. 471-480.

Kosala, R. and Blockeel, H. (2000), 'Web mining research: a survey', SIGKDD Explorations 2(1), 1-15.

Kruschwitz, U., Webb, N. and Sutcliffe, R. (2008), Query Log Analysis for Adaptive DialogueDriven Search, Query Log Analysis, Information Science Reference, 389-414, chapter XX.

Mobasher, B., Cooley, R. and Srivastava, J. (2000), 'Automatic personalization based on web usage mining', Commmunications of the ACM 43(8), 142-150.

Nasraoui, O., Soliman, M., Saka, E., Badia, A. and Germain, R. (2008), 'A web usage mining framework for mining evolving user profiles in dynamic web sites', IEEE Transactions on Knowledge and Data Engineering 20(2), 202-215.

Pazzani, M. J. and Billsus, D. (2007), Content-Based Recommendation Systems, Vol. 4321 of Adaptation Technologies, Springer-Verlag Berlin Heidelberg 2007, 325-341, chapter II.

Perkowitz, M. and Etzioni, O. (1997), 'Adaptive web sites: an ai challenge', Artificial Intelligence 11(1), 246-271.

Perkowitz, M. and Etzioni, O. (1999), 'Adaptive web sites: Conceptual cluster mining', Artificial Intelligence 17(1), 243-273.

Perkowitz, M. and Etzioni, O. (2000), 'Towards adaptive web sites: Conceptual framework and case study', Artificial Intelligence 118(1), 245-275.

Schafer, J., Frankowski, D., Herlocker, J. and Sen, S. (2007), Collaborative Filtering Recommender Systems, Vol. 4321 of Adaptation Technologies, Springer-Verlag Berlin Heidelberg 2007, 291324, chapter II.

Srivastava, J., Cooley, R., Deshpande, M. and Tan, P.-N. (2000), 'Web usage mining: Discovery and applications of usage patterns from web data', SIGKDD Explorations 1(2), 12-23.

Teevan, J., Dumais, S. T. and Horvitz, E. (2005), 'Beyond the commons: Investigating the value of personalizing web search', User Modeling and User-Adapted Interaction 13(1), 311-372. 